\title{
Atypical deep peroneal neuropathy In presence of accessory deep peroneal nerve
}

\author{
L. GUTMANN
}

\begin{abstract}
From the Department of Neurology, West Virginia University Medical Center, Morgantown, West Virginia, U.S.A.
\end{abstract}

SUMMARY The clinical and electrophysiological findings in two patients with a deep peroneal neuropathy occurring in the presence of significant innervation of the extensor digitorum brevis muscle by the accessory deep peroneal nerve are reported. This nerve is a branch of the superficial peroneal nerve which was not involved in either cases reported. An awareness of this anatomical variation of innervation of the extensor digitorum brevis muscle is important for the correct clinical and electromyographic evaluations of deep peroneal nerve lesions. The electromyographic findings in this situation are sufficiently characteristic to identify clearly this anatomical variant. From a clinical point of view, residual function in the lateral portion of the extensor digitorum brevis muscle, in the face of an otherwise complete deep peroneal palsy, should suggest this anatomical variation.

The accessory deep peroneal nerve is a common anatomical variant innervating the extensor digitorum brevis muscle of the foot. The innervation of this muscle is usually exclusively via the deep peroneal nerve. However, in as many as $28 \%$ of patients one or both of the extensor digitorum brevis muscles are partially innervated by the accessory deep peroneal nerve, a branch of the superficial peroneal nerve (Winckler, 1934; Lambert, 1969; Infante and Kennedy, 1970).

An awareness of this anomalous innervation is important especially in the clinical and electromyographic evaluation of deep peroneal neuropathies if incorrect conclusions are to be avoided. It is the purpose of this paper to stress the clinical and electromyographic characteristics of deep peroneal - neuropathy in the presence of the accessory deep peroneal nerve innervating the extensor digitorum brevis muscle.

\section{METHODS}

Nerves were stimulated with a single rectangular electrical pulse from a Grass S-4 stimulator. The common peroneal nerve was stimulated at the knee above the head of the fibula, the deep peroneal nerve at the dorsum of the ankle, and the accessory deep peroneal nerve over the postero-lateral aspect of the ankle, just behind the lateral malleolus (Fig. 1). Nerves were stimulated supramaximally at all sites using percutaneous stimulating electrodes.

The surface recording electrode was placed over the mid-point of the extensor digitorum brevis muscle and a reference electrode over the base of the fifth toe. Motor unit action potentials and spontaneous activity were recorded with monopolar teflon coated electrodes with a reference electrode applied to the skin.

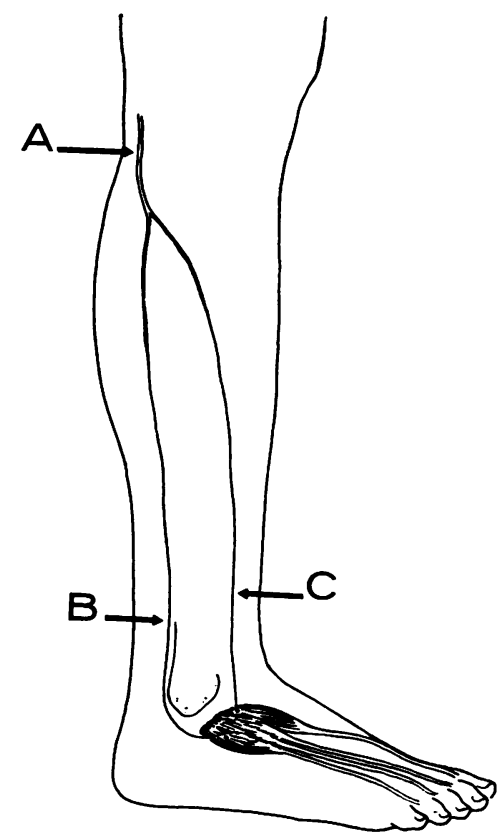

FIG. 1. Nerves stimulated in this study: (A) common peroneal nerve; (B) accessory deep peroneal nerve (branch of superficial peroneal nerve); and (C) deep peroneal nerve. 
The action potentials were amplified and displayed using Tektronix amplifier Type 3A74, differential amplifier Type 2A61, time base Type 2B67, and cathode ray oscilloscope Type 561A. Data were recorded on Kodak TRI-X Pan 35 mm. film.

\section{CASE 1}

(WVU H19-95-65) In 1965 this 19-year-old girl suddenly developed a painless left foot drop which showed no significant subsequent progression.

Examination in November 1968 showed a complete left foot drop with atrophy of the anterior tibialis and extensor hallucis longus muscles. The ability to dorsiflex slightly the fourth and fifth digits of the left foot was retained. Visible contraction of the extensor digitorum brevis muscle was limited to the most lateral aspect of the muscle. Inversion, eversion, and plantar flexion of the left foot were performed normally.

A benign expanding lesion was noted at the proximal end of the left fibula on radiographic examination. In December 1968 the proximal end of the fibula containing an osteochondroma was removed uneventfully. The deep peroneal nerve was noted to be stretched over the mass in the proximal portion of the fibula.

Examination in December 1969 was similar to that noted one year earlier.

\section{CASE 2}

(WVU H21-45-68) This 56-year-old man contused the lower one-third of his tibia in 1959 after which he had intermittent episodes of pain and swelling in this area and his ankle once or twice each year. These always cleared spontaneously. He had several such episodes after June 1969. During such an episode a sudden and spontaneous right foot drop developed.

Examination four weeks later (October 1969) showed a complete paralysis of the right anterior tibialis and extensor hallucis longus muscles. Contraction in the extensor digitorum brevis was limited to the lateral aspect. Ability to dorsiflex the fourth and fifth toes was retained. Posterior tibialis, peronei, gastrocnemius and the intrinsic muscles of the right foot functioned normally.

Radiographs of the right tibia and fibula failed to show any bony lesions. A ganglion cyst, compressing the deep peroneal nerve below the head of the fibula, was removed surgically. Examination in December 1969 showed no clinical change.

\section{RESULTS}

ELECTROMYOGRAPHIC STUDY CASE I Stimulation of the left common peroneal nerve at the knee produced a twitch of the lateral aspect of the extensor digitorum brevis muscle and a muscle action potential $4.6 \mathrm{mV}$ in amplitude. No response was obtained from the anterior tibialis muscles. Stimulation of the left deep peroneal nerve over the dorsum of the ankle failed to evoke either a twitch or muscle action potential. Stimulation of the accessory deep peroneal nerve on the postero-lateral aspect of the ankle (immediately posterior to the lateral malleolusy evoked a similar twitch and muscle action potentie from the extensor digitorum brevis as stimulation of the common peroneal nerve at the knee (Fig. 2f Motor conduction velocity in the common peroneat -accessory deep peroneal nerve-was $38.4 \mathrm{~m}$ pe second.

$A$

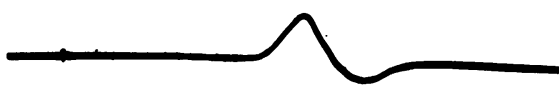

B

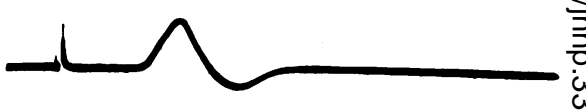

$\omega$

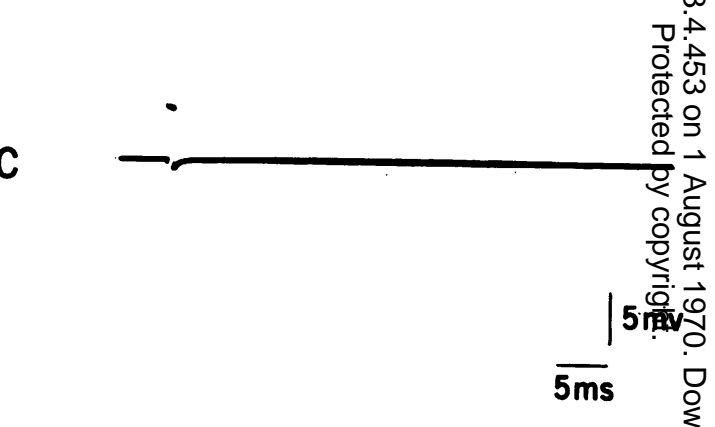

FIG. 2. Case 1. Muscle action potentials evoked from the left extensor digitorum brevis muscle on stimulatio of $(A)$ common peroneal nerve; (B) accessory deep peronea nerve; and (C) deep peroneal nerve. Note no potentiat was evoked on stimulating $\mathbf{C}$.

Needle electromyographic examination of the left anterior tibialis and extensor hallucis longus muscles showed a mild number of fibrillations ang no motor unit potentials under voluntary controb In the left extensor digitorum brevis no fibrillation were noted and there was a moderate decrease i the number of motor unit potentials present. These fired rapidly and showed an increased proportion of large units up to $12 \mathrm{mV}$ in amplitude. Examination of the left peroneus longus and posterior tibialis muscles was normal.

ELECTROMYOGRAPHIC STUDY CASE 2 Stimulation off the right common peroneal nerve at the knee evoked a muscle twitch from the lateral portion of the 
extensor digitorum brevis and none from the anterior tibialis muscle. Evoked muscle action potential from extensor digitorum brevis was $3.8 \mathrm{mV}$ in amplitude. Stimula:ion of the right deep peroneal nerve over the dorsum of the ankle produced only a very small muscle action potential $0.1 \mathrm{mV}$ in amplitude. Stimulation of the accessory deep peroneal nerve on the postero-lateral aspect of the right ankle (immediately posterior to the lateral malleolus) evoked a twitch and a muscle action potential from the extensor digitorum brevis similar to that noted on stimulation of the right common peroneal nerve. The evoked muscle action potential $(3.6 \mathrm{mV})$ was, however, slightly smaller (Fig. 3). Motor conduction velocity in the right common peroneal-accessory deep peroneal nerve-was $42 \cdot 4 \mathrm{~m}$ per second.

A

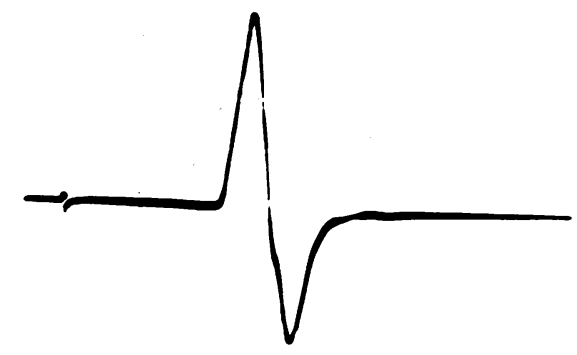

B

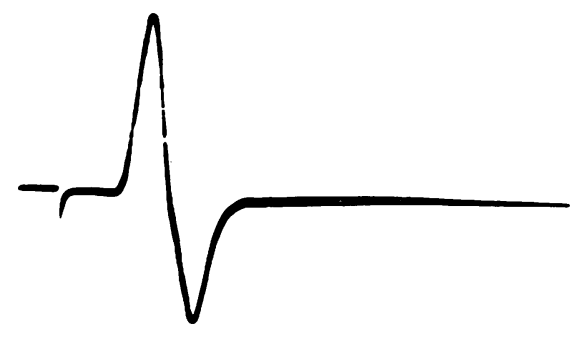

C

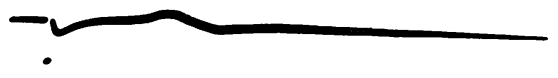

$\operatorname{lmv}$

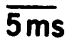

FIG. 3. Case 2. Muscle action potentials evoked from the right extensor digitorum brevis muscle on stimulation of (A) common peroneal nerve; (B) accessory deep peroneal nerve; and (C) deep peroneal nerve. Note only very small potential evoked on stimulating $\mathrm{C}$.

Examination of the medial aspect of the right extensor digitorum brevis showed a few fibrillations and no motor unit potentials under voluntary control. Examination of the lateral aspect of the extensor digitorum brevis showed a few fibrillations with motor unit potentials present although they were moderately decreased in number firing rapidly. Large motor unit action potentials up to $10 \mathrm{mV}$ in amplitude were noted. Many fibrillations and no motor unit potentials under voluntary control were noted in the right anterior tibialis and extensor hallucis longus muscles. Examination of the peroneus longus and posterior tibialis muscles was normal.

\section{DISCUSSION}

The clinical and electrophysiological findings clearly indicated the presence of a deep peroneal neuropathy in both patients. Clinically, marked weakness was present only in muscles innervated by the deep peroneal nerve with complete sparing of muscles innervated by the superficial peroneal nerve. The only unusual and unexpected finding was the relatively good function of the lateral portion of the extensor digitorum brevis muscle. In case 1 , this initially led to the incorrect conclusion that the deep peroneal neuropathy was only a partial one. In case 2 , however, the initial interpretation was correctthat is, that the functioning lateral portion of the extensor digitorum brevis in face of complete weakness of other muscles innervated by the deep peroneal nerves was, in fact, due to innervation by the accessory deep peroneal nerve.

Needle electromyographic examination substantiated the clinical evaluation of the distribution of involved muscles. Fibrillations and loss of all motor unit potentials under voluntary control were limited to muscles innervated by the deep peroneal nerve without involvement of muscles innervated by the superficial peroneal nerve. In both patients only partial involvement of extensor digitorum brevis muscles was noted because of the partial innervation by the accessory deep peroneal nerve.

In the involved leg of both patients, stimulation of the common peroneal nerve at the knee evoked a muscle action potential from the extensor digitorum brevis along with a visible twitch from the lateral portion of this muscle. At the same time there was no twitch visible from the anterior tibialis muscle. In case 1 the identical responses from the extensor digitorum brevis were produced by stimulating the accessory deep peroneal nerve behind the lateral malleolus, while no response was evoked when the deep peroneal nerve on the dorsum of the ankle was stimulated. In case 2 the identical findings were noted with the exception that a very small evoked muscle action potential was produced when the deep peroneal nerve was stimulated at the ankle indicating that in this patient most, but not all, of the remaining innervation to the extensor digitorum 
brevis was mediated by the accessory deep peroneal nerve.

The observation that the accessory deep peroneal nerve innervates a portion of the extensor digitorum brevis muscle unilaterally or bilaterally in as many as $28 \%$ of patients (or $22 \%$ of legs) certainly indicates that this is a common anatomical variant (Winckler, 1934; Lambert, 1969; Infante and Kennedy, 1970). An awareness of this is important if one is to avoid erroneous conclusions regarding a complete deep peroneal neuropathy. The presence of residual clinical function and voluntary motor unit action potentials in the lateral portion of the extensor digitorum brevis muscle may be misinterpreted as representing a partial deep peroneal neuropathy. As in the cases presented, the conduction studies clearly documented that these findings were due to innervation by the accessory deep peroneal nerve. The electromyographic and conduction study changes in the normal individual may also be confusing in the presence of this anatomical variant if one is not aware of it (Lambert, 1969).

The findings noted in these two patients support the previous physiological (Lambert, 1969; Infante and Kennedy, 1970) and anatomical (Bryce, 1896; Winckler, 1934) studies which concluded that the accessory deep peroneal nerve was a motor branch of the superficial peroneal nerve and that it innervates the lateral portion of the extensor digitorum brevis. In both patients the superficial peroneal nerve was spared on both clinical and electromyographic examination and all or almost all the residual innervation of the extensor digitorum brevis muscle was through the accessory deep peroneal nerve.

\section{REFERENCES}

Bryce, T. H. (1896). Long muscular branch of the musculocutaneous nerve of the leg. J. Anat., 31, V-XII.

Infante, E., and Kennedy, W. R. (1970). Anomalous branch of the peroneal nerve detected by electromyography. Arch. Neurol., 22, 162-165.

Lambert, E. H. (1969). The accessory deep peroneal nerve: a common variation in innervation of extensor digitorum brevis. Neurology (Minneap.), 19, 1169-1176.

Winckler, G. (1934). Le nerf péronier accessoire profond: étude d'anatomie comparée. Arch. Anat. Histol. Embryol., 18, 181-219. 